\title{
CCL19/CCL21-triggered signal transduction and migration of dendritic cells requires prostaglandin E2
}

Elke Scandella, Ying Men, Daniel F. Legler, Silke Gillessen, Ladislav Prikler, Burkhard Ludewig, and Marcus Groettrup

The control of dendritic cell (DC) migration is pivotal for the initiation of cellular immune responses. When activated with inflammatory stimuli, the chemokine receptor CCR7 is up-regulated on DCs. Activated DCs home to lymphoid organs, where the CCR7 ligands CCL19 and CCL21 are expressed. We previously found that human monocyte-derived DCs (MoDCs) exclusively migrated to CCL19 and CCL21 when matured in the presence of prostaglandin (PG) E2. Because PGE2 did not alter CCR7 cell surface expression, we examined whether PGE2 may exert its effect by coupling CCR7 to signal transduction modules. Indeed, stimulation with CCR7 ligands led to enhanced phosphatidylinositol-3-kinase-mediated phosphorylation of protein kinase $B$ when MoDCs were matured in the presence of PGE2. Moreover, CCL19/CCL21-induced intracellular calcium mobilization in MoDCs occurred only when PGE2 was present during maturation. MoDC migra- tion to CCL19 and CCL21 was dependent on phospholipase $C$ and intracellular calcium flux but not on phosphatidylinositol-3 kinase. Hence, our data provide insight into CCL19/CCL21-triggered signal transduction pathways and identify a novel function for PGE2 in controlling the migration of mature MoDCs by facilitating CCR7 signal transduction. (Blood. 2004; 103:1595-1601)

\section{Introduction}

Dendritic cells (DCs) are professional antigen-presenting cells capable of antigen transport and presentation in secondary lymphoid organs, which is crucial for the initiation and maintenance of T-cell-mediated immune responses. ${ }^{1}$ They reside in the periphery in an immature state, taking up pathogens through pinocytosis or receptor-mediated endocytosis, leading to the induction of DC maturation. Mature DCs undergo phenotypical and functional changes, including up-regulation of the chemokine receptor CCR7. ${ }^{2}$ The expression of CCR7 on mature DCs and on naive and central memory $\mathrm{T}$ cells is essential for their coordinated migration to the T-cell area of draining lymph nodes because this migration is guided by CCL19 (EBI1-ligand chemokine [ELC], macrophage inflammatory protein [MIP]-3ß), and CCL21 (secondary lymphoidtissue chemokine [SLC], 6-Ckine), the 2 ligands for CCR7. Both chemokines are expressed by stromal cells in the T-cell area of secondary lymphoid organs. The essential role of CCR7 and its ligands for the migration of mature DCs to lymph nodes was demonstrated in CCR7-deficient mice ${ }^{3}$ and plt/plt mice, which lack the ligands for CCR7. ${ }^{4}$

Chemokine receptors are transmembrane receptors of the class A rhodopsin-like family, which span the membrane 7-fold and transduce their signals through $G$ proteins, mainly of the $G_{\alpha i}$ subclass. ${ }^{5}$ Receptor stimulation leads to the inhibition of adenyl cyclases through $\mathrm{G}_{\alpha \mathrm{i}}{ }^{-}$and $\mathrm{G}_{\beta \gamma^{-}}$-mediated activation of phospholipase C (PLC), followed by diacylglycerol (DAG)-mediated activation of protein kinase $\mathrm{C}$ (PKC) and the release of calcium from intracellular stores. ${ }^{6}$ Furthermore, $G_{\beta \gamma}$ subunits that are released from $G_{\alpha i}$ proteins transiently activate phosphatidylinositol 3-kinases (PI3Ks), leading to the activation of protein kinase $\mathrm{B}$ (PKB, Akt) and extracellular signal-regulated kinase-2 (Erk-2). ${ }^{7}$ However, most data on the signal transduction of chemokine receptors have been obtained with CXCR1, CCR2, CCR5, and CXCR4, but little information is available about CCR7 signal transduction. Recently, Rho-associated kinases have been shown to be required for the CCR7-mediated polarization and chemotaxis of T lymphocytes. ${ }^{8}$ Another study suggests that CCR7 signaling involves the activation of a Janus kinase (JAK). ${ }^{9}$ It is still a matter of debate, however, whether the JAK/signal transducer and activator of transcription (JAK/STAT) pathway, which requires chemokine receptor dimerization, contributes to chemokine-mediated signal transduction. ${ }^{10}$ In addition, most studies have been performed with neutrophils, peritoneal macrophages, and T cells but not with DCs.

Recently, we and others observed that the maturation-induced up-regulation of CCR7 expression on human monocyte-derived DCs (MoDCs) was insufficient to allow MoDC migration to CCL19 and CCL21. ${ }^{11,12}$ Human MoDCs matured either with soluble CD40L (sCD40L) or with polyI:C markedly enhanced surface expression of CCR7 but were not at all or were only poorly responsive to CCL19 and CCL21. Interestingly, MoDC migration to CCL19 and CCL21 was readily observed on maturation in the presence of the proinflammatory mediator prostaglandin E2 (PGE2), though PGE2 did not change the expression level of CCR7 on mature MoDCs, providing evidence for an alternative effect of 
PGE2. The importance of PGE2 for DC migration in vivo has recently been shown in Ptger $4^{-/-}$mice lacking the PGE2 receptor EP4. These mice display impaired migration of Langerhans cells and reduced skin immune responses. ${ }^{13}$ However, other DC types, such as $\mathrm{CD} 1 \mathrm{~b} / \mathrm{c}^{+}$peripheral blood $\mathrm{DCs}$, do not require an additional PGE2 stimulus for effective CCR7-mediated migration. ${ }^{12}$

In this study we investigated the hypothesis that PGE2 may facilitate CCL19/CCL21-directed migration by coupling the cognate chemokine receptor CCR7 to its signal transduction modules. Indeed, we found that PGE2 was required during sCD40Lstimulated maturation of MoDCs to activate PKB and to mobilize intracellular free calcium as downstream signaling events of CCR7. Moreover, we demonstrate that PLC-mediated cytoplasmic $\mathrm{Ca}^{2+}$ mobilization was a prerequisite for the chemotaxis of MoDCs to CCL19 and CCL21. Our results, hence, provide evidence that PGE2 is required to facilitate the CCR7-mediated signal transduction and migration of MoDCs.

\section{Materials and methods}

\section{Tissue culture media and reagents}

AIM V was purchased from Invitrogen (Groningen, The Netherlands). Interleukin-4 (IL-4) was obtained from Strathmann (Hamburg, Germany), and granulocyte macrophage-colony-stimulating factor (GM-CSF) was obtained from Novartis (Leukomax, Basel, Switzerland). Soluble CD40L (sCD40L) was kindly provided by Immunex (Seattle, WA). Prostaglandin E2 was purchased from Pharmacia \& Upjohn (Prostin E2; Dübendorf, Switzerland). Human CCL19 and CCL21 were provided by R\&D Systems (Wiesbaden-Nordenstadt, Germany), and CXCL-12 was purchased from PromoCell (Heidelberg, Germany). Staurosporine, U-73122, H-89, wortmannin, Ly-294002, Y-27632 and 1,2-bis(o-aminophenoxy)ethane- $N, N, N^{\prime}, N^{\prime}-$ tetraacetic acid-tetra(acetomethyl) ester (BAPTA-AM) were purchased from Calbiochem (Lucerne, Switzerland). Phorbol-myristate-acetate (PMA) and ionomycin was purchased from Sigma (Buchs, Switzerland).

\section{Antibodies}

Horseradish peroxidase (HRP)-conjugated antimouse antibody was obtained from DAKO (Zug, Switzerland), and antirabbit antibody was obtained from Milan (La Roche, Switzerland). Antibodies against Akt/ PKB, phospho-Akt/PKB (Ser473), p42/44 mitogen-activated protein (MAP) kinase/Erk-1/2, and phospho-p44/42 MAP kinase/Erk-1/2 (Thr202/Tyr204) were from Cell Signaling Technology (Frankfurt, Germany).

\section{Generation of human MoDCs}

MoDCs were generated from human peripheral blood mononuclear cells (PBMCs), as described previously. ${ }^{11}$ Briefly, PBMCs were separated by density gradient centrifugation on Ficoll-Paque (Pharmacia, Uppsala, Sweden), resuspended at $4 \times 10^{6}$ cells $/ \mathrm{mL}$ in AIM V medium, and allowed to adhere to plastic for 1 hour at $37 \mathrm{C}$. Nonadherent cells were removed, and remaining cells were cultured in AIM V medium supplemented with GM-CSF $(50 \mathrm{ng} / \mathrm{mL})$ and IL-4 (1000 U/mL). MoDCs derived from the adherence step were only used when the content of remaining B and T cells was below 2\%. Alternatively, monocytes were purified from PBMCs by positive selection using anti-CD14-conjugated magnetic microbeads (Miltenyi Biotec, Bergisch Gladbach, Germany) and were cultivated at $1 \times 10^{6}$ cells/mL. On day 6 , nonadherent and loosely adherent cells were harvested and recultured $\left(5 \times 10^{5}\right.$ cells $\left./ \mathrm{mL}\right)$ for an additional 48 hours in cytokinecontaining medium in the absence or presence of $1 \mu \mathrm{g} / \mathrm{mL}$ sCD40L to induce maturation. Where indicated, PGE2 $(1 \mu \mathrm{g} / \mathrm{mL})$ or $\mathrm{H}-89$ was added during stimulation.

\section{Cell stimulation and Western blot analysis}

MoDCs were washed twice with phosphate-buffered saline (PBS) and resuspended in AIM V medium without additives $\left(2 \times 10^{7}\right.$ cells $\left./ \mathrm{mL}\right)$.
Aliquots of $2 \times 10^{6}$ cells were incubated for 10 minutes at $37 \mathrm{C}$ and were stimulated with CCL19 or CCL21 (250 ng/mL) for different periods of time. Incubations were terminated by the addition of trichloroacetic acid (TCA) to a final concentration of $10 \%$. Protein pellets were washed twice with ice-cold acetone and dissolved by boiling in $1 \times$ Laemmli sodium dodecyl sulphate (SDS) loading buffer containing 5\% 2-mercaptoethanol. Proteins were separated on $10 \%$ SDS-polyacrylamide gel electrophoresis (SDS-PAGE) and were transferred to nitrocellulose membranes. The membranes were blocked with 5\% low-fat dry milk in $1 \times$ TBS-buffer and were incubated with the respective antibodies overnight on a rocking plate at $4 \mathrm{C}$. After washing, HRP-conjugated secondary antibodies were bound and detected using enhanced chemiluminescence (Pierce, Socochim, Lausanne, Switzerland). Thereafter, membranes were stripped with $0.2 \mathrm{M}$ $\mathrm{NaOH}$ for 5 minutes and then washed and reprobed with control antibodies.

\section{Chemotaxis assay}

Chemotaxis of MoDCs was measured by migration through a polycarbonate filter of $5 \mu \mathrm{m}$ pore size in 24-well transwell chambers (Corning Costar, Cambridge, MA). AIM V $(600 \mu \mathrm{L})$ containing indicated doses of CCL19, CCL21, CXCL-12, or medium alone as a control for spontaneous migration was added to the lower chamber; $1 \times 10^{5} \mathrm{DCs}(100 \mu \mathrm{L})$ were added to the upper chamber and were incubated for 3 hours at 37 C. A 500- $\mu \mathrm{L}$ aliquot of the cells that migrated to the bottom chamber was counted by flow cytometry in a FACScan acquiring events for a fixed time period of 60 seconds using CellQuest software (Becton Dickinson, Basel, Switzerland). Each experiment was performed in duplicate. The mean number of spontaneously migrated cells was subtracted from the total number of migrated cells. Values are given as percentage of migrated cells \pm SEM. Where indicated, cells were incubated for 30 minutes at $37 \mathrm{C}$ with various drugs and were washed twice before the migration assay.

\section{Intracellular free $\mathrm{Ca}^{2+}$ mobilization}

MoDCs $\left(1 \times 10^{6} / \mathrm{mL}\right)$ were loaded with $4 \mu \mathrm{M}$ fluo-3-actetomethylester (fluo-3-AM) in the presence of $1 \mu \mathrm{M}$ pluronic F-127 (Molecular Probes, Leiden, The Netherlands) in loading-buffer (145 mM NaCl, $5 \mathrm{mM} \mathrm{KCl,} 1$ $\mathrm{mM} \mathrm{Na} \mathrm{HPO}_{4}, 1 \mathrm{mM} \mathrm{MgCl} 2,5 \mathrm{mM}$ glucose, $1 \mathrm{mM} \mathrm{CaCl}_{2}$, and $10 \mathrm{mM}$ HEPES ( $N$-2-hydroxyethylpiperazine- $N$ '-2-ethanesulfonic acid; $\mathrm{pH}$ 7.5) for 30 minutes at room temperature. Where indicated, BAPTA-AM was loaded simultaneously into MoDCs. Subsequently, cells were washed twice with loading buffer or $\mathrm{Ca}^{2+}$-free loading buffer containing 2 mM EGTA (ethylene glycol-bis(2-aminoethylether)- $N, N, N^{\prime}, N^{\prime}$-tetraacetic acid) and were stored on ice. For aquisition, cells were incubated for 10 minutes at 37 C before stimulation with CCL19, CCL21 (250 ng/mL), or ionomycin ( $1 \mu \mathrm{g} / \mathrm{mL})$, and fluorescence was recorded over time by flow cytometry.

\section{Results}

\section{MoDC migration to CCL19 and CCL21 requires activation of protein kinase A and Rho-associated kinases}

Previously, we and others demonstrated that human MoDCs required PGE2 during maturation to migrate to CCL19 and CCL21 effectively. ${ }^{11,12}$ Earlier studies showing PGE2-induced migration of MoDCs were performed in the presence of high PGE2 concentrations (in the micromolar range), at concentrations far above the physiologic levels found in extracellular fluids at least under noninflammatory conditions. ${ }^{14}$ For a better understanding of the effect of PGE2 to enhance MoDC migration, we first performed transwell chemotaxis assays with MoDCs matured with sCD40L and graded concentrations of PGE2. Maturation with CD40L resulted in mature MoDCs with homogeneous surface expression of CCR7 irrespective of the addition of PGE2 (Figure 1A). ${ }^{11}$ However, CCL19- and CCL21-induced migration of SCD40Lmatured MoDCs required PGE2 and were dose dependent and 
A

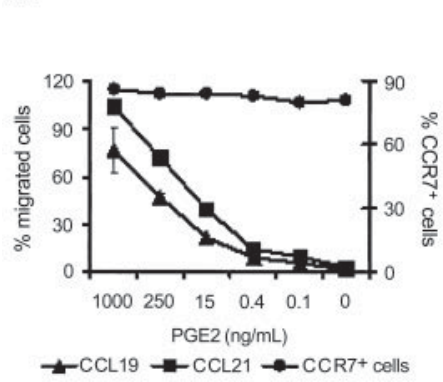

B

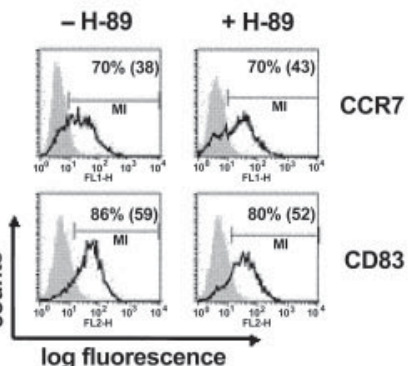

C

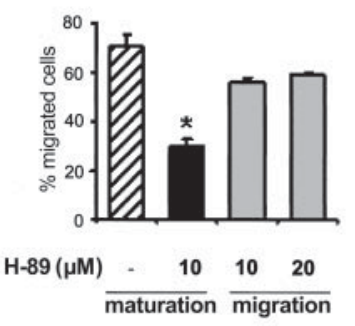

D

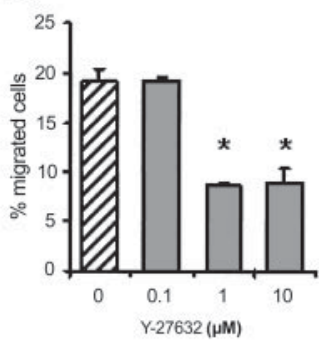

Figure 1. PGE2-induced MoDC migration to CCL19 and CCL21 is PKA and Rho kinase dependent. (A) Titration of PGE2; immature MoDCs were stimulated wi h sCD40L and titrated concentrations of PGE2. Subsequently, MoDCs were tested for CCL19- and CCL21-triggered migration in transwell assays (left axis) and were analyzed by flow cytometry for the expression of CCR7 (right axis). (B) MoDCs were matured with SCD40L and PGE2 in the presence or absence of the PKA inhibitor H-89 for 48 hours. Subsequen ly, MoDCs were analyzed by flow cytometry for the expression of CD83 and CCR7. The portion of gated positive cells is given as a percentage, with the mean

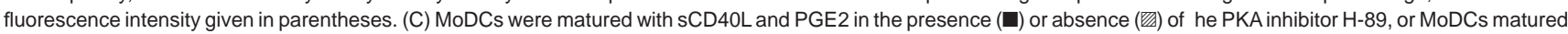
with SCD40L and PGE2 were preincubated with H-89 for 30 minutes (四) before they were allowed to migrate in response to CCL21. (D) MoDCs matured wi h SCD40L and PGE2 were left untreated (遂) or were pretreated with the Rho kinase inhibitor Y-27632 (圆) and were analyzed in a migra ion assay in response to CCL21. Results from 1 of 3 representative experiments are shown. Error bars indicate SEM and asterisks indicate a significant reduction.

already significant at a PGE2 concentration of $15 \mathrm{ng} / \mathrm{mL}$ (Figure 1A). These PGE2 concentrations were expected to occur at sites of inflammation but not in healthy tissues. Furthermore, PGE2 was needed throughout the MoDC maturation process because 1-hour preincubation of mature MoDCs with PGE2 was insufficient to induce MoDC migration in response to CCL19 and CCL21 (data not shown).

PGE2 signaling occurs through the prostaglandin receptors EP2 and EP4, which are expressed on MoDCs and which lead to increased cyclic adenosine monophosphate (cAMP) levels and to the activation of cAMP-dependent protein kinase A (PKA) in the cytosol. ${ }^{11}$ Therefore, we investigated whether MoDC migration in the presence of PGE2 is dependent on the activation of PKA. To this end, MoDCs were stimulated with sCD40L and PGE2 in the presence of the PKA-specific inhibitor $\mathrm{H}-89$. Treating MoDCs with H-89 at a concentration of $10 \mu \mathrm{M}$ had no effect on MoDC maturation, CCR7 expression, or viability (Figure 1B and data not shown). However, adding $\mathrm{H}-89$ for the entire period of maturation reduced the migration of MoDCs in response to CCL19 and CCL21 by approximately $50 \%$ (Figure 1C). In contrast, blocking activation of PKA during the migration assay had no significant effect on MoDC migration, indicating that the PGE2-induced activation of PKA during MoDC maturation may provide a signal that allows them to migrate.

Recently, the Rho family of GTPases was shown to be involved in CCR7-mediated migration of $\mathrm{T}$ lymphocytes. ${ }^{8}$ To test the involvement of Rho kinase, mature MoDCs were pretreated with graded doses of Y-27632, a specific inhibitor of Rho-associated kinases. Blocking Rho-associated kinases resulted in a significant reduction of CCL21-mediated migration of MoDCs (Figure 1D), confirming the findings observed in T lymphocytes.

\section{PGE2 does not affect chemokine-mediated Erk-1/2 activation}

Because PGE2 did not alter the cell surface expression of CCR7 on sCD40L-matured MoDCs, we investigated whether PGE2 might facilitate migration by coupling CCR7 to its signal transduction pathway. Signal transduction of CCR7 in DCs has not been addressed so far. An earlier study on T cells, however, demonstrated that CCL19 stimulation resulted in the activation of the MAP-kinase cascade, leading to a transient activation of Erk-2 by phosphorylation at threonine and tyrosine residues. ${ }^{7}$ We matured MoDCs with sCD40L in the presence or absence of PGE2, stimulated them with CCL19 for different time periods, and investigated Erk activation using Western blotting. Using an antibody detecting Erk-1 and Erk-2 exclusively when phosphorylated at Thr202 and Tyr204, we found rapid and transient Erk-1/2 activation after 2 to 7 minutes of CCL19 stimulation (Figure 2A). However, there was no significant difference in Erk-1/2 activation or duration between MoDCs matured with sCD40L in the presence or absence of PGE2.

\section{PGE2 enhances CCL19-mediated PKB activation}

A second major pathway triggered by chemokines is the stimulation of phosphatidyl-inositol 3-kinase (PI3-kinase) leading to the formation of phosphatidyl-3,4,5,-trisphosphate (PIP3) and the activation of PKB. ${ }^{7}$ Hence, we investigated the activation of PKB in MoDCs on CCR7 triggering. CCL19 induced rapid and transient phosphorylation of PKB in MoDCs, as demonstrated by Western blotting using phospho Ser473-specific PKB antibodies (Figure 2B). Interestingly, PKB activation was stronger and more prolonged in MoDCs matured in the presence of PGE2 than in MoDCs matured with sCD40L alone. PKB phosphorylation peaked after 2 minutes of CCL19 triggering and remained detectable after 15
A

Figure 2. CCL21-mediated activation of Erk-1/2 and PKB in MoDCs. MoDCs were matured in he presence or absence of PGE2 and subsequently were stimulated with CCL21 $(250 \mathrm{ng} / \mathrm{mL})$ for the indicated time periods. Whole-cell lysates were separated on SDS-PAGE and transferred to nitrocellulose membranes. (A) Membranes were stained with an antibody against phosphorylated (Thr202/Tyr204) Erk-1/2 (p-Erk) or total Erk-1/2 (t-Erk). (B) Membranes were probed with an antibody against phosphorylated (S473) PKB (p-PKB) or total PKB (t-PKB). Results from 1 of 4 independent representative experiments are shown.

p-Erk
t-Erk
p-Erk
t-Erk

B
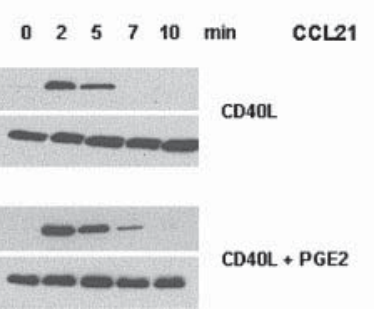
CD40L CL21

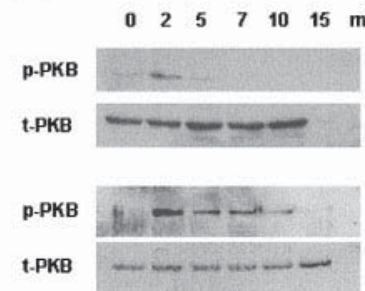
$\mathrm{CD} 40 \mathrm{~L}+\mathrm{PGE2}$ CD40L 
A

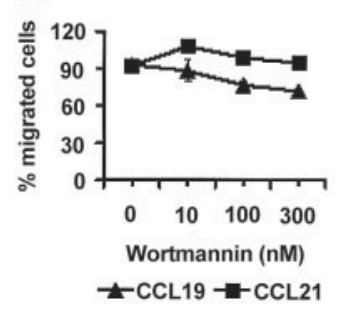

B

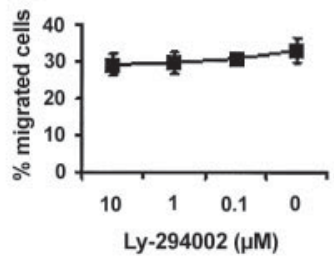

C

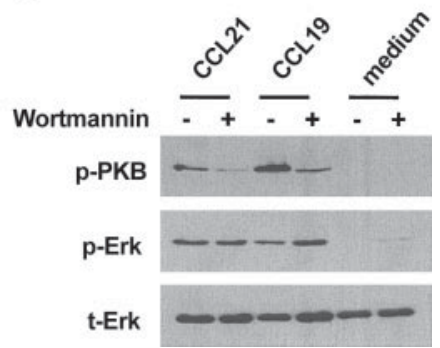

Figure 3. Activation of PKB is not required for the migration of MoDCs in response to CCL19 and CCL21. MoDCs matured in the presence of PGE2 were incubated for 30 minutes with graded doses of (A) wortmannin or (B) Ly-294002 and were tested for migration to CCL21 and CCL19 in a transwell assay. Error bars indicate SEM. (C) To confirm the effec iveness of wortmannin, MoDCs treated with or without wortmannin were stimulated with CCL19 or CCL21 for 2 minutes. Whole-cell lysates were separated on SDS-PAGE, and activated PKB or Erk-1/2 was detected with an antibody against PKB phosphorylated at S473 (p-PKB) or Erk-1/2 dually phosphorylated at Thr202 and Tyr204 (p-Erk). Membranes were reprobed with an antibody reacting with total Erk-1/2 (t-Erk) to confirm equal protein loading. Results from 1 of 3 representative experiments using different MoDC preparations are shown.

minutes in MoDCs matured in the presence of PGE2. In contrast, MoDC maturation without PGE2 allowed only weak phosphorylation of PKB after 2 minutes, which disappeared after 5 minutes of chemokine stimulation (Figure 2B). In some experiments, the activation of PKB in MoDCs matured in the absence of PGE2 was even below the detection limit (data not shown).

\section{Migration of MoDCs to CCL19 and CCL21 is not dependent on PKB activation}

We next analyzed whether inefficient chemokine-mediated activation of PKB in MoDCs matured in the absence of PGE2 was responsible for their reduced migration. Hence, MoDCs matured in the presence of PGE2 were incubated for 10 minutes with the specific PI3-kinase inhibitor wortmannin or Ly-249002, each of which blocks the downstream activation of PKB. Unexpectedly, neither wortmannin nor Ly-249002 had an effect on MoDC migration to CCL19 and CCL21 (Figure 3A-B). To rule out the ineffective inhibition of PKB phosphorylation by wortmannin, MoDC lysates were subjected to Western blot analysis. Preincubating MoDCs with $100 \mathrm{nM}$ wortmannin prevented Ser473 phosphorylation of PKB almost completely, but Erk-1/2 activation was not affected by this treatment (Figure 3C), indicating that wortmannin inhibition was effective.

\section{PGE2 is required for CCL19/CCL21-mediated $\mathrm{Ca}^{2+}$ mobilization}

Another consequence of chemokine receptor stimulation is the activation of G-protein-sensitive PLC isoforms, resulting in the generation of DAG and inositol 3,4,5-triphosphate, which leads to the release of $\mathrm{Ca}^{2+}$ from intracellular stores. Hence, we analyzed CCL19/CCL21-induced $\mathrm{Ca}^{2+}$ mobilization in MoDCs matured in the presence or absence of PGE2 by flow cytometry. Triggering MoDCs with CCL19 or CCL21 resulted in a rapid rise in intracellular free $\mathrm{Ca}^{2+}$ concentrations only on maturation in the presence (Figure 4C-D), but not in the absence, of PGE2 (Figure $4 \mathrm{~A}-\mathrm{B}$ ). $\mathrm{Ca}^{2+}$ mobilization was substantially reduced when PGE2treated MoDCs were preincubated with the specific PLC inhibitor U73122 (Figure 5C). In contrast, increases in cytosolic free $\mathrm{Ca}^{2+}$ concentrations were less affected by the chelation of extracellular $\mathrm{Ca}^{2+}$ concentrations by 2 mM EGTA (Figure 5B). The combination of both - the inhibition of PLC with U73122 and the presence of 2 mM EGTA-abrogated the $\mathrm{Ca}^{2+}$ signal completely (Figure 5D), indicating that intracellular and extracellular calcium stores contributed to the cytoplasmic calcium flux. MoDC migration in response to CCL21 was not reduced in the presence of $2 \mathrm{mM}$ EGTA in the assay buffer (Figure 6A), which suggests that calcium mobilization from intracellular stores suffices to maintain the migratory capacity.

\section{PLC activation is required for MoDC migration to CCL19, CCL21, and CXCL-12}

To assess whether the activation of PLC is required for migration, MoDCs were incubated with increasing amounts of the PLC inhibitor U73122. As depicted in Figure 6B, MoDC migration in response to CCL19 and CCL21 was completely blocked after treatment with $200 \mathrm{nM}$ U73122. Half-maximal inhibition was observed at $50 \mathrm{nM}$ U73122.

$\mathrm{Ca}^{2+}$ activates, in conjunction with DAG, various isoforms of PKC. Additionally, PKC can be activated through signal transduction events initiated by the stimulation of the PI3-kinase pathway. The contribution of PKC to MoDC migration was investigated using either the PKC inhibitor staurosporine or the PKC activator
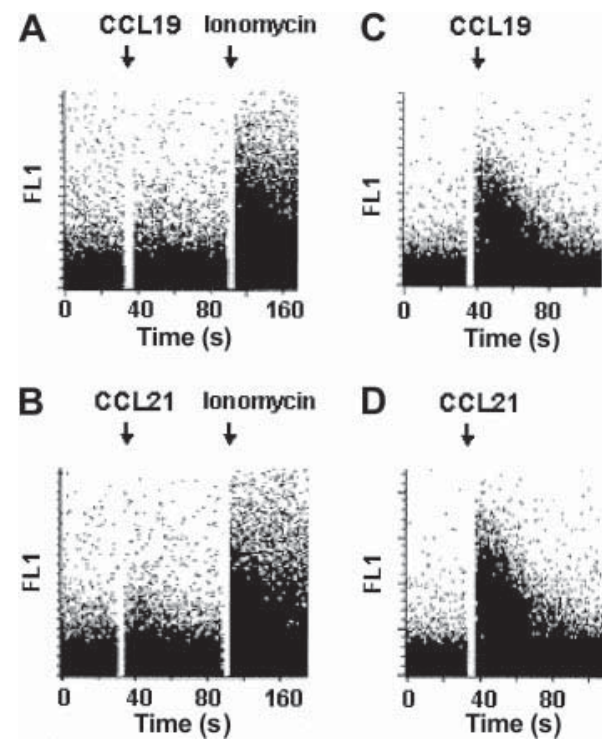

Figure 4. Chemokine-induced $\mathrm{Ca}^{2+}$ mobilization differs in MoDCs matured in the presence or absence of PGE2. MoDCs stimulated with SCD40L in he absence (A-B) or presence (C-D) of PGE2 were loaded with fluo-3-AM, and chemokineinduced $\mathrm{Ca}^{2+}$ mobiliza ion was analyzed by flow cytometry. Baseline was established for 30 seconds before chemokines $(250 \mathrm{ng} / \mathrm{mL})$ were added, as indicated by the arrow. Ionomycin $(1 \mu \mathrm{g} / \mathrm{mL})$ was added (A-B) to ensure proper fluo-3-AM loading of MoDCs. Representative results obtained from MoDCs of a single donor are shown. At least 5 independent experiments with MoDCs from different donors yielded similar results. 

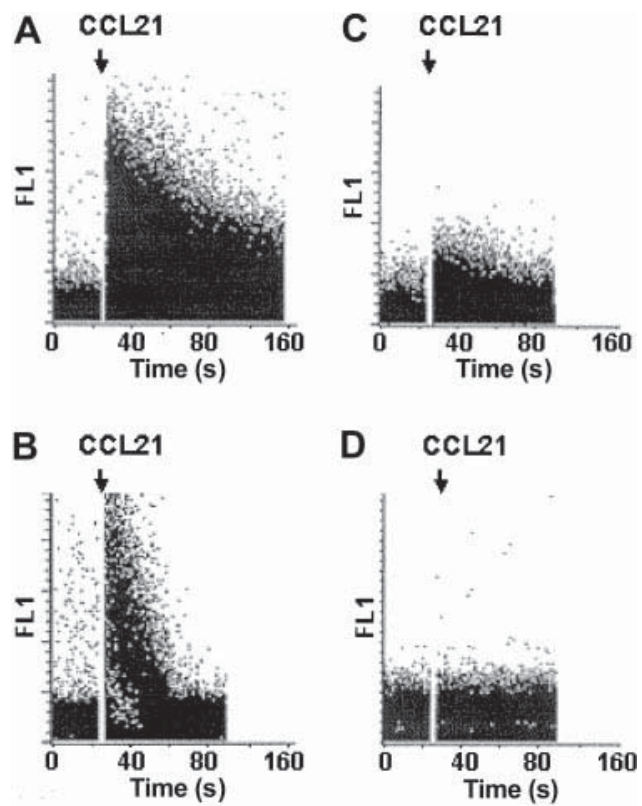

Figure 5. CCL21 mediates $\mathrm{Ca}^{2+}$ mobilization from intracellular stores. MoDCs stimulated with SCD40L and PGE2 were loaded with fluo-3-AM, and chemokineinduced $\mathrm{Ca}^{2+}$ mobiliza ion was analyzed using flow cytometry (A). Experiments (B-D) were performed in $\mathrm{Ca}^{2+}$-free buffer in the presence of $2 \mathrm{mM} \mathrm{EGTA.} \mathrm{(C-D)} \mathrm{The} \mathrm{release}$ of $\mathrm{Ca}^{2+}$ from intracellular stores was inhibited by the addi ion of the PLC inhibitor U73122 $(10 \mu \mathrm{M})$. Baseline was established for 30 seconds before CCL21 (250 $\mathrm{ng} / \mathrm{mL}$ ) was added, as indicated by he arrow. Representative results obtained from MoDCs of a single donor are shown. At least 3 independent experiments with MoDCs from different donors gave similar results.

PMA. Staurosporine is an unspecific protein kinase inhibitor that inhibits PKC ( $\left.\mathrm{IC}_{50}, 3 \mathrm{nM}\right)$, PKA ( $\left.\mathrm{IC}_{50}, 7 \mathrm{nM}\right)$, and $\mathrm{Ca}^{2+} /$ calmodulin- $^{2}$ dependent (CaM) kinase 2 ( $\left.\mathrm{IC}_{50}, 20 \mathrm{nM}\right){ }^{15,16}$ Pretreatment of MoDCs matured in the presence of PGE2 with staurosporine at effective concentrations to block PKC (4 nM) did not affect MoDC migration to CCL19 and CCL21. However, staurosporine significantly reduced the migration of MoDCs in response to CCL19 and CCL21 at concentrations that interfered with the activation of other protein kinases such as CaM kinases (Figure 6D). Interestingly, the migration of MoDCs to CCL19 and CCL21 was abrogated after pretreatment with graded concentrations of PMA, implicating that the heterologous activation of PKC impedes chemokine-induced signaling (Figure 6C).

To investigate whether the dependence of MoDC migration on PGE2 was valid for another chemokine receptor of MoDCs, we examined the CXCR4-directed migration of MoDCs to SDF-1/
CXCL12 (Figure 6E). Remarkably, the migration of MoDCs to CXCL12 in transwell assays occurred only when MoDCs were matured in the presence of PGE2, in agreement with a recent report by Luft et al. ${ }^{12}$ In addition, migration to CXCL12 was inhibited with the PLC inhibitor U73122 but not with the PI3K inhibitor wortmannin.

\section{Intracellular $\mathrm{Ca}^{2+}$ flux is required for MoDC migration}

To test whether $\mathrm{Ca}^{2+}$ mobilization is essential for migration, the cell-permeable $\mathrm{Ca}^{2+}$ chelator BAPTA-AM was used to trap liberated $\mathrm{Ca}^{2+}$ in the cytoplasm after chemokine stimulation of PGE2treated MoDCs (Figure 7B). Chelation of intracellular free $\mathrm{Ca}^{2+}$ by BAPTA-AM inhibited the migration of MoDCs to CCL19 and CCL21 in a dose-dependent manner (Figure 7A), thus indicating that $\mathrm{Ca}^{2+}$ mobilization is required for MoDC migration. Taken together, MoDCs require PGE2 during maturation for CCL19/ CCL21-induced activation of $\mathrm{PKB}$ and $\mathrm{Ca}^{2+}$ mobilization. In contrast to the PI3-kinase-mediated activation of PKB, activating the PLC pathway leading to the liberation of intracellular free $\mathrm{Ca}^{2+}$ is essential for MoDC migration.

\section{Discussion}

Up-regulation of the chemokine receptor CCR7 on DCs is crucial for their homing to secondary lymphoid organs, where the CCR7 ligands CCL19 and CCL21 are expressed. Two recent studies demonstrated that CCR7, though present on mature MoDCs, failed under certain conditions to mediate migration to CCL19 and CCL21, suggesting that CCR7 can exist on the cell surface in an inactive form. ${ }^{11,12}$ One of the factors required for MoDC migration to lymph node-derived chemokines was identified as the inflammatory mediator PGE2, which transmits its effect through the elevation of intracellular cAMP. As we have demonstrated, the amount of PGE2 and the duration of PGE2 stimulation during maturation seemed to be critical for CCR7-mediated MoDC migration. PGE2 concentrations at inflammatory sites were reported to be between $0.2 \mathrm{nM}$ and $1.69 \mu \mathrm{M} .{ }^{14,17,18}$ Here, we demonstrate that at a physiologic concentration of PGE2, MoDCs are capable of migrating in response to CCL19 and CCL21. It is also likely that at sites of inflammation, the DC microenvironment contains high levels of PGE2 because it is surrounded by other immune cells, such as monocytes, that produce large amounts of this mediator once they are stimulated. ${ }^{19}$
A

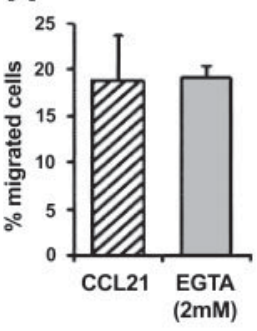

B

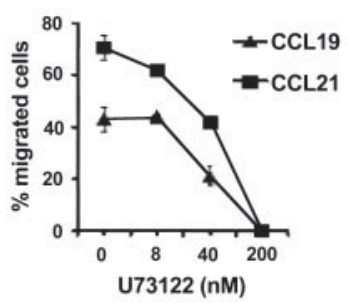

C

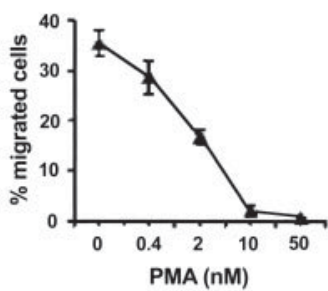

D

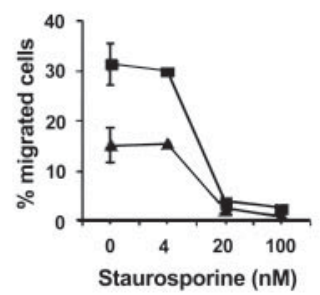

E

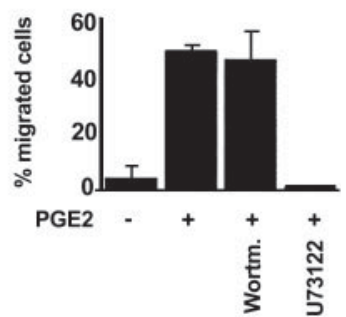

Figure 6. PLC pathway is involved in MoDC migration to CCL19, CCL21, and CXCL12. (A) MoDCs matured with SCD40L and PGE2 were analyzed in a migra ion assay in

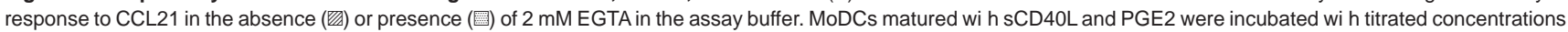
of (B) he PLC inhibitor U73122, (C) the PKC activator PMA, or (D) the PKC inhibitor staurosporine for 30 minutes at $37^{\circ} \mathrm{C}$ before migration to CCL19 and CCL21was analyzed in a transwell chemotaxis assay. (E) Migration to CXCL12 of MoDCs matured in he presence or absence of PGE2. The PI3K inhibitor wortmannin was used at $100 \mathrm{nM}$, and U73122 was used at $10 \mu \mathrm{M}$. Results from representative experiments of at least 3 different MoDC preparations are shown. Error bars indicate SEM. 
A

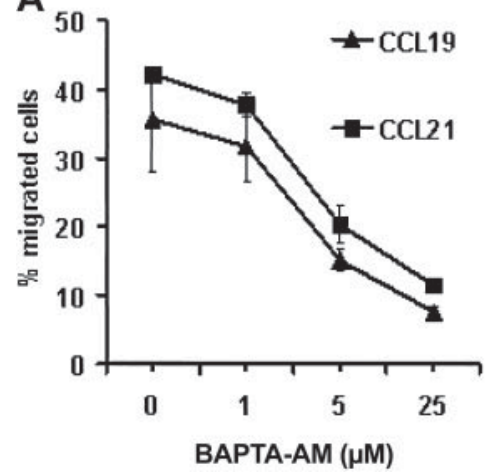

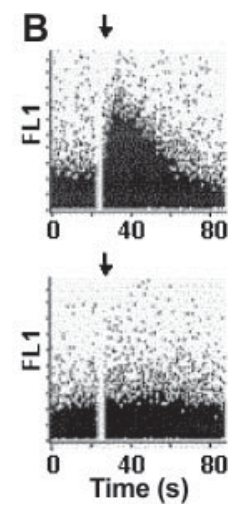

Figure 7. Chemokine-induced $\mathrm{Ca}^{2+}$ mobilization is required for the migration of MoDCs to CCL19 and CCL21. (A) SCD40L- and PGE2-stimulated MoDCs were incubated with graded doses of BAPTA-AM for 30 minutes at room temperature before migration to CCL19 and CCL21 was analyzed in a transwell chemotaxis assay. Error bars indicate SEM. (B) SCD4OL- and PGE2-stimulated MoDCs were loaded with fluo-3-AM alone (top) or with BAPTA-AM (bottom). Chemokine-induced $\mathrm{Ca}^{2+}$ mobilizaion was analyzed by flow cytometry. Baseline was established for 30 seconds before CCL21 was added, as indicated by he arrow. Representative results obtained from MoDCs of a single donor are shown. Three independent experiments with MoDCs from different donors yielded similar results.

Increased cytosolic cAMP levels after PGE2 stimulation and subsequent activation of the cAMP-dependent PKA seem to play a key role in activating the migratory capacity of MoDCs. We showed that inhibiting cAMP-dependent PKA dramatically reduces MoDC chemokine-directed migration. Furthermore, a recent study shows that stimulating MoDCs with adenosine triphosphate (ATP), which also leads to increased intracellular cAMP levels, enhances the migration of MoDCs to CCR7 ligands. ${ }^{20}$

CCL19/CCL21-induced migration of MoDCs is pertussis toxin sensitive, indicating that the responding chemokine receptor, CCR7, is coupled to $G$ proteins of the $G_{\alpha i}$ type. ${ }^{11}$ In this study we show that CCR7 stimulation with CCL19 and CCL21 involves the activation of at least 3 main pathways in PGE2treated MoDCs, including PI3-kinase and PKB phosphorylation, PLC activation and calcium release, and the MAP-kinase pathway. The requirement for PI3-kinase and PLC activation in chemokine-induced migration is still a matter of debate and seems to vary with the cell type and with the kind of chemokine receptor analyzed. Although the migration of human neutrophils to formyl peptides, complement components such as C5a or C3a, and CXCL8 (IL-8) was independent of PI3-kinase activation, studies in mice lacking the PI3-kinase- $\gamma$ isoform showed reduced neutrophil migration to these chemoattractants. ${ }^{21-23}$ In addition it has been reported that, in contrast to monocytes, the migration of human T lymphocytes to CCL5 was sensitive to the specific PI3-kinase inhibitor wortmannin. ${ }^{24}$ We show that MoDC chemotaxis to CCL19 and CCL21 is independent of the activation of PI3-kinase because migration was insensitive to the PI3-kinase inhibitors wortmannin and Ly294002. This is in accordance with a recent report that treating freshly isolated mouse lymphocytes with wortmannin or Ly294002 had only minor effects on lymphocyte chemotaxis to CCL $21 .^{9}$

The requirement of PLC activation for chemotaxis was mainly reported for murine neutrophils. Neutrophils from PLC- $\beta 2$ - and PLC- $\beta 3$-deficient mice were normal with respect to chemotaxis to formyl peptides or CXCL8, ${ }^{21}$ indicating that the $\mathrm{Ca}^{2+}$ signal is not elementary for chemotaxis in these cells. MoDC migration to CCL19 and CCL21, in contrast, was strictly dependent on the activation of PLC and the liberation of intracellular free $\mathrm{Ca}^{2+}$, as shown in our experiments with the

PLC-specific inhibitor U73122 and by chelating intracellular $\mathrm{Ca}^{2+}$. One possible role of chemokine-induced $\mathrm{Ca}^{2+}$ mobilization in MoDCs could be the activation of $\mathrm{Ca}^{2+} /$ calmodulindependent kinases (CaMKs), as reported for CXCL8-mediated migration of human neutrophils. ${ }^{25}$ Consistent with this notion, treating MoDCs with staurosporine at concentrations that affect CaMK activation significantly reduced the migration of MoDCs to CCL19 and CCL21 (Figure 6D).

Interestingly, chemokine-induced activation of Erk-1/2 was similar in MoDCs matured in the presence or absence of PGE2, suggesting that MAP-kinase activation does not mediate CCL19 or CCL21 migration of MoDCs. Indeed, there is not much evidence that Erk activation is required for cell migration, as shown for neutrophils and monocytes. ${ }^{26,27}$ Although Erk activation relies on $\mathrm{G}$ proteins, the chemokine receptor or cell type determines whether Erk-1/2 phosphorylation occurs directly through G-protein-activated ras or an indirect pathway involving the activation of PI3-kinase and the subsequent stimulation of ras through PKC. The involvement of tyrosine kinase in CCR7-mediated chemotaxis has been shown by Stein et $\mathrm{al}^{9}$ for primary lymphocytes using the JAK inhibitor tyrphostin (AG490). We also observed partial inhibition of MoDC migration to CCL21 using the same concentration $(100 \mu \mathrm{M})$ of AG490, but the outcome of these experiments was too variable to make a definitive statement (data not shown).

Our results clearly show that PGE2 is essential for CCR7mediated $\mathrm{Ca}^{2+}$ mobilization and for the subsequent migration of MoDCs. Thus, we provide evidence that CCR7 can act as a functional decoy receptor on MoDCs in the absence of PGE2. The expression of chemokine receptors that fail to generate active intracellular signaling after ligand engagement has been reported in several cell types. For instance, inflammatory chemokine receptors on monocytes and MoDCs treated with IL-10 in combination with an inflammatory stimulus are not down-regulated, but they fail to respond to their respective ligands. ${ }^{28}$ Moreover, germinal center $\mathrm{B}$ cells are unable to activate chemotaxis in response to CXCL12 in spite of their surface expression of CXCR4. ${ }^{29}$

Chemokine receptor inactivation can occur through phosphorylation of the receptor itself or the downstream effector PLC- $\beta{ }^{30}$ Accordingly, receptor inactivation was mediated by heterologous activation of PKC after ligand binding to other chemoattractant receptors; hence, this mechanism is referred to as cross-desensitization. The fact that MoDC migration to CCL19 and CCL21 can be inhibited through heterologous activation of PKC by PMA (Figure 6C) or ATP ${ }^{20}$ suggests that CCR7 may be inactivated by cross-desensitization. Correspondingly, CXCR4 inactivation and monocyte migration failure in response to CCL3 (MIP-1 $\alpha$ ), CCL5, formyl peptides, and CCL2 (monocyte chemoattractant protein [MCP]-1) were reported after PMA treatment. ${ }^{30}$ However, other mechanisms for chemokine receptor inactivation, such as up-regulation of the suppressors of cytokine signaling proteins that were shown to bind to CXCR4 and to block JAK/STAT and $G_{\alpha i}$ pathways, have been described. ${ }^{31}$

In conclusion, we show that MoDCs require environmental instruction by PGE2 to couple CCR7 expression to signal transduction pathways and migration in vitro. Given also that CXCR4-mediated migration of MoDCs to CXCL-12 depended on the presence of PGE2 during maturation, this phenomenon of receptor coupling may be valid for other chemokine receptors (Figure 6E). ${ }^{12}$ This puts a new focus onto PGE2 and its role in 
the regulation of immune responses. ${ }^{13}$ We provide evidence that CCL19- and CCL21-induced migration of MoDCs require activation of the PLC pathway and liberation of intracellular free $\mathrm{Ca}^{2+}$ but are independent of PI3-kinase activation and subsequent phosphorylation of PKB. These findings could open up therapeutic opportunities for immune diseases and transplantation medicine and for the development of MoDCbased vaccines.

\section{Acknowledgments}

We thank Immunex Corporation for providing sCD40L and Wolfhart Seelentag, Hans Schiefer, and Markus Arn for the irradiation of cells. We thank Dr Markus Fopp and the personnel of St Gallen blood bank for supplying blood products and Edith Uetz von Allmen for help with some of the experiments.

\section{References}

1. Banchereau J, Steinman RM. Dendritic cells and he control of immunity. Nature. 1998:392:245252.

2. Sallusto F, Schaerli P, Loetscher P, et al. Rapid and coordinated switch in chemokine receptor expression during dendritic cell maturation. Eur J Immunol. 1998;28:2760-2769.

3. Förster R, Schubel A, Breitfeld D, et al. CCR7 coordinates the primary immune response by establishing functional microenvironments in secondary lymphoid organs. Cell. 1999;99:23-33.

4. Luther SA, Tang HL, Hyman PL, Farr AG, Cyster JG. Coexpression of the chemokines ELC and SLC by $T$ zone stromal cells and deletion of the ELC gene in the plt/plt mouse. Proc Natl Acad Sci U S A. 2000;97:12694-12699.

5. Mellado M, Rodriguez-Frade JM, Manes S, Marinze-A C. Chemokine signalling and functiona reponses: the role of receptor dimerization and TK pathway activation. Annu Rev Immunol. 2001 19:397-421.

6. Wu D, Huang $\mathrm{CK}$, Jiang $\mathrm{H}$. Roles of phospholipid signaling in chemoattractant-induced responses. J Cell Sci. 2000;113:2935-2940.

7. Tilton B, Ho L, Oberlin E, et al. Signal transducion by $C X C$ chemokine receptor 4 : stromal cellderived factor 1 stimulates prolonged protein kinase $B$ and extracellular signal-regulated kinase 2 activation in T lymphocytes. J Exp Med. 2000; 192:313-324.

8. Bardi G, Niggli V, Loetscher P. Rho kinase is required for CCR7-mediated polariza ion and chemotaxis of T lymphocytes. FEBS Lett. 2003;542: 79-83.

9. Stein JV, Soriano SF, M'rini C, et al. CCR7-mediated physiological lymphocyte homing involves ac iva ion of a tyrosine kinase pathway. Blood. 2003;101:38-44

10. Thelen M, Baggiolini M. Is dimerization of chemokine receptors functionally relevant? Sci STKE. 2001;2001(PE34):1-2.

11. Scandella E, Men Y, Gillessen S, Förster R, Groettrup M. Prostaglandin E2 is a key factor for CCR7 surface expression and migra ion of monocyte-derived dendritic cells. Blood. 2002;100: 1354-1361.

12. Luft $\mathrm{T}$, Jefford $\mathrm{M}$, Luetjens $\mathrm{P}$, et al. Functionally distinct dendri ic cell (DC) populations induced by physiologic stimuli: prostaglandin E2 regulates he migratory capacity of specific DC subsets. Blood. 2002;100:1362-1372.

13. Kabashima K, Sakata D, Nagamachi M, Miyachi Y, Inaba K, Narumiya S. Prostaglandin E2-EP4 signaling initiates skin immune responses by promoting migration and maturation of Langerhans cells. Nat Med. 2003;9:744-749.

14. Anderson GD, Hauser SD, McGarity KL, Bremer $M E$, Isakson PC, Gregory SA. Selective inhibi ion of cyclooxygenase (COX)-2 reverses inflammaion and expression of COX-2 and interleukin 6 in rat adjuvant arthritis. J Clin Invest. 1996;97:26722679.

15. Ruegg UT, Burgess GM. Staurosporine, K-252 and UCN-01: potent but nonspecific inhibitors of protein kinases. Trends Pharmacol Sci. 1989;10: 218-220.

16. Yanagihara N, Tachikawa E, Izumi F, Yasugawa S, Yamamoto H, Miyamoto E. Staurosporine: an effec ive inhibitor for Ca2+/calmodulin-dependent protein kinase II. J Neurochem. 1991;56: 294-298.

17. Hirata I, Murano M, Nitta M, et al. Estima ion of mucosal inflammatory mediators in rat DSS-induced colitis: possible role of PGE(2) in protecion against mucosal damage. Digestion. 2001; 63(suppl 1):73-80.

18. Hinson RM, Williams JA, Shacter E. Elevated interleukin 6 is induced by prostaglandin E2 in a murine model of inflammation: possible role of cyclooxygenase-2. Proc Natl Acad Sci U S A. 1996;93:4885-4890.

19. Penglis PS, Cleland LG, Demasi M, Caughey GE, James MJ. Differential regulation of prostaglandin E2 and thromboxane A2 production in human monocytes: implications for the use of cyclooxygenase inhibitors. J Immunol. 2000;165: 1605-1611.

20. Schnurr M, Toy T, Stoitzner P, et al. ATP gradients inhibit the migratory capacity of specific human dendritic cell types: implications for P2Y11 receptor signaling. Blood. 2003;102:613-620.

21. Li Z, Jiang H, Xie W, Zhang Z, Smrcka AV, Wu D. Roles of PLC- $\beta 2$ and $-\beta 3$ and PI3K $\gamma$ in chemoattractant-mediated signal transduction. Science. 2000;287:1046-1049.
22. Hirsch E, Katanaev VL, Garlanda C, et al. Central role for $\mathrm{G}$ protein-coupled phosphoinositide 3-kinase gamma in inflammation. Science. 2000;287: 1049-1053.

23. Sasaki T, Irie-Sasaki J, Jones RG, et al. Function of PI3K $\gamma$ in thymocyte development, T cell activaion, and neutrophil migration. Science. 2000; 287:1040-1046.

24. Turner SJ, Domin J, Waterfield MD, Ward SG, Westwick J. The CC chemokine monocyte chemotactic peptide-1 activates both the class I p85/ p110 phosphatidylinositol 3-kinase and the class II PI3K-C2 $\alpha$. J Biol Chem. 1998;273:2598725995.

25. Verploegen $\mathrm{S}$, van Leeuwen CM, van Deutekom HW, Lammers JW, Koenderman L, Coffer PJ. Role of $\mathrm{Ca}_{2}+$ /calmodulin regulated signaling pathways in chemoattractant induced neutrophil effector functions: comparison wi h he role of phospho idylinositol-3 kinase. Eur J Biochem. 2002;269:4625-4634.

26. Cambien B, Pomeranz M, Millet MA, Rossi B, Schmid-Alliana A. Signal transduc ion involved in MCP-1-mediated monocy ic transendo helial migration. Blood. 2001;97:359-366.

27. Knall C, Wor hen GS, Johnson GL. Interleukin 8-stimulated phospha idylinositol-3-kinase ac ivity regulates the migration of human neutrophils independent of extracellular signal-regulated kinase and p38 mitogen-activated protein kinases. Proc Natl Acad Sci U S A. 1997;94:3052-3057.

28. D'Amico G, Frascaroli G, Bianchi G, et al. Uncoupling of inflammatory chemokine receptors by IL-10: generation of func ional decoys. Nat Immunol. 2000;1:387-391.

29. Bleul CC, Schultze JL, Springer TA. B lymphocyte chemotaxis regulated in associa ion with microanatomic localization, differentia ion state, and B cell receptor engagement. J Exp Med. 1998;187: 753-762.

30. Ali H, Richardson RM, Haribabu B, Snyderman R. Chemoattractant receptor cross-desensitizaion. J Biol Chem. 1999;274:6027-6030.

31. Soriano SF, Hernanz-Falcon P, Rodriguez-Frade $\mathrm{JM}$, et al. Functional inactivation of CXC chemokine receptor 4-mediated responses through SOCS3 up-regulation. J Exp Med. 2002;196:311321. 